\title{
Causes of uveitis in children without juvenile idiopathic arthritis
}

This article was published in the following Dove Press journal:

Clinical Ophthalmology

25 June 2015

Number of times this article has been viewed

\section{Stephanie B Engelhard \\ Asima Bajwa \\ Ashvini K Reddy}

Department of Ophthalmology, University of Virginia, Charlottesville, VA, USA
Correspondence: Ashvini K Reddy Department of Ophthalmology, University of Virginia, 1300 Jefferson Park Avenue, Charlottesville, VA 22908, USA

Tel + I 4342435890

Fax + I 4349245180

Email akr5u@hscmail.mcc.virginia.edu
Background: The purpose of this study was to report the demographics, disease characteristics, treatments, and visual outcomes of pediatric uveitis patients without juvenile idiopathic arthritis managed in a tertiary medical center.

Methods: A retrospective, observational study was performed in pediatric uveitis patients without juvenile idiopathic arthritis and aged 0-18 years, who were seen at the University of Virginia from 1984 to 2014.

Results: Thirty-nine pediatric uveitis patients (57 eyes) were identified. The patient population was 51.28\% female, 51.28\% Caucasian, and 33.33\% African American. The mean age at diagnosis was 11.9 years. The mean duration of follow-up was 3.11 years. The mean number of visits to the clinic was 10.41 . Of 57 eyes, 31 (54.39\%) had anterior uveitis, $12(21.05 \%)$ had intermediate uveitis, nine $(15.79 \%)$ had posterior uveitis, and five $(8.77 \%)$ had panuveitis. The leading diagnoses were traumatic uveitis (25.64\%), undifferentiated anterior uveitis $(17.95 \%)$, undifferentiated intermediate uveitis (15.38\%), HLA-B27-associated anterior uveitis $(7.69 \%)$, and herpetic anterior uveitis (7.69\%). Systemic associations included sarcoidosis, ulcerative colitis, and psoriatic arthritis $(n=3)$. The most common treatment modalities included local steroids $(66.67 \%)$, systemic steroids $(23.08 \%)$, and antimetabolites $(20.51 \%)$. Ocular hypertension was found in five $(12.82 \%)$ patients. Ocular surgery was performed in six $(15.38 \%)$ patients. Mean best-corrected visual acuity (BCVA) at baseline across all anatomical locations was 0.458 $\log$ MAR, and was $0.411 \log$ MAR at final follow-up. Mean BCVA improved during follow-up in all but the anterior uveitis group. The mean baseline intraocular pressure was $14.27 \mathrm{mmHg}$, and was $14.22 \mathrm{mmHg}$ at final follow-up.

Conclusion: Uveitis in childhood is a vision-threatening group of inflammatory disorders arising from numerous etiologies that vary geographically and historically. Because of the high burden of disease, the difficulty of making precise etiologic diagnoses, and the complicated management, it is imperative that affected children be referred to and closely monitored by uveitis specialists to prevent devastating ocular damage. This study found that BCVA and intraocular pressure did not vary significantly during follow-up, suggesting that close management by an ophthalmologist may prevent adverse visual outcomes, and highlighted the high prevalence of traumatic uveitis in children, which tends to have good visual outcomes.

Keywords: uveitis, pediatric, visual acuity, intraocular pressure

\section{Introduction}

Uveitis describes a heterogeneous group of inflammatory diseases of the eye representing both systemic immune-mediated and infectious processes as well as inflammatory processes localized to the eye. Although children represent only $2.1 \%-13.8 \%$ of patients seen in uveitis clinics, they can present significant diagnostic and therapeutic challenges. ${ }^{1-3}$ Children with uveitis are often preverbal or asymptomatic, and may present to the ophthalmologist with already significant ocular damage., ${ }^{4,5}$ 
Furthermore, the scope of possible etiologies of childhood uveitis is quite vast and often differs from that in the adult population. The etiologic and anatomical distribution of uveitis has also been shown to vary considerably with geographic location and throughout history. ${ }^{2}$

Some previous studies report an increase in severe vision loss in children with uveitis compared to adult patients, ${ }^{6-8}$ a finding that has long-term implications for quality of life and future productivity. In spite of the cited disease aggressiveness in children, ophthalmologists may be reticent to prescribe long-term corticosteroid treatment or surgical intervention for fear of increased risk of undesirable side effects. ${ }^{5,7}$ In addition to these diagnostic and treatment challenges, the uveitis classification scheme often varies from clinic to clinic. Although efforts have been made to standardize the uveitis nomenclature, most notably by the Standardization of Uveitis Nomenclature Working Group, ${ }^{9}$ discrepancies still exist in uveitis classification and diagnosis. This retrospective, observational study was performed to report the etiologies of uveitis in the pediatric uveitis population at a Mid-Atlantic US tertiary referral center and to analyze the demographics, diagnoses, treatment modalities, outcomes, and complications in this population.

\section{Materials and methods}

This was a retrospective, observational study of all pediatric uveitis patients seen in the Department of Ophthalmology at the University of Virginia. The study was approved by the institutional review board and was conducted in accordance with the principles of the Declaration of Helsinki. A database of all patients with a diagnosis of uveitis is maintained by the Department of Ophthalmology at the University of Virginia. The database was reviewed to identify patients diagnosed with uveitis and aged 0-18 years over a 30-year period from 1984 through 2014. Thirty-nine pediatric uveitis patients were identified from the database. No minimum follow-up period was required for inclusion, and all data in the database were collected from patient charts completed at the time of patient visit. Time points for data collection in the database included each patient's initial visit to the clinic (baseline) and final follow-up visit. Disease classification was recorded in accordance with the Standardization of Uveitis Nomenclature Working Group criteria. ${ }^{9}$ As there were variable practice and referral patterns in our area over the period of this study, a decision was made to exclude patients with juvenile idiopathic arthritis (JIA) from analysis to avoid inconsistencies.
The database was reviewed for patient demographic information, including date of birth, sex, race/ethnicity, age at presentation, duration of follow-up, and number of clinic visits. Clinical information, including final diagnosis, location, laterality, and chronicity of uveitis, best-corrected visual acuity (BCVA), intraocular pressure (IOP), relevant systemic and associated diagnoses, treatment modalities, and complications, was also reviewed. BCVA results were converted to logarithm of the minimum angle of resolution (logMAR) units for analysis and are given as $\log$ MAR (mean \pm standard deviation). Visual acuity of counting fingers was recorded as 2.0 logMAR; hand movements, 2.3 logMAR; light perception, $2.6 \log$ MAR; and no light perception, $2.9 \operatorname{logMAR} .^{10,11}$ Uveitis etiologies were confirmed by imaging with optical coherence tomography, fluorescein angiography, and automated perimetry, and by further ancillary testing including serology, radiology, microbiology, and biopsy when appropriate. Intraocular infection was confirmed with fluid sampling or biopsy for microscopy and cytology and culture or polymerase chain reaction, when appropriate. Post-procedural uveitis was defined as ocular inflammation following intraocular surgery, laser, or intravitreal injection. In cases where the cause of uveitis could not be identified by the above techniques, the term "undifferentiated" was ascribed. Management including the use of local and systemic steroids, antihypertensive drops, intravitreal injections, subtenon injections, antimetabolites, anti-tumor necrosis factor agents, cataract surgery, pars plana vitrectomy, and glaucoma management (medical and surgical intervention) were recorded. Given the size of the data set, descriptive statistical analysis was performed.

\section{Results}

Thirty-nine patients (57 eyes) diagnosed with uveitis and aged 0-18 years were identified and included in the study. Of the 39 patients, 20 (51.28\%) were female, 20 (51.28\%) were Caucasian, 13 (33.33\%) were African American, $2(5.13 \%)$ were Hispanic, and four $(10.26 \%)$ were of another race. The mean ( \pm standard deviation) age at diagnosis of uveitis was $11.9 \pm 4.39$ years, and the mean age at presentation to the ophthalmology clinic at the University of Virginia was 13.2 \pm 5.01 years. The mean duration of follow-up was $3.1 \pm 5.72$ years. The total follow-up duration ranged from 1 day to 30 years. The mean number of visits to the ophthalmology clinic was $10.41 \pm 10.36$ (range $1-43$ ). The anterior uveitis group had the youngest age of onset at $11.0 \pm 4.62$ years, the youngest age at presentation at $12.0 \pm 4.69$ years, the highest mean duration of follow-up at $3.76 \pm 6.84$ years, and 
the greatest number of visits to the clinic at $11.71 \pm 12.03$. The posterior uveitis group had the oldest age of both diagnosis and presentation at $13.7 \pm 3.07$ years and 17.0 \pm 2.73 years, respectively. This group also had the lowest mean duration of follow-up at $0.96 \pm 1.19$ years. Patient demographic information and characteristics by anatomical location are summarized in Table 1.

Thirty-one eyes of 24 patients were diagnosed as having anterior uveitis, which was the most common anatomical location. Twelve eyes of seven patients were diagnosed with intermediate uveitis, nine eyes of five patients were diagnosed with posterior uveitis, and five eyes of three patients were diagnosed with panuveitis. The distribution of uveitis by anatomical location is summarized in Table 2.

The most common pediatric uveitis diagnosis in our population was uveitis secondary to trauma $(n=10,25.64 \%)$. Other common diagnoses included undifferentiated anterior uveitis $(n=7,17.95 \%)$, undifferentiated intermediate uveitis $(\mathrm{n}=6,15.38 \%)$, HLA-B27-associated anterior uveitis $(\mathrm{n}=3$, $7.69 \%)$, herpetic anterior uveitis $(n=3,7.69 \%)$, undifferentiated panuveitis $(n=2,5.13 \%)$, and tubulointerstitial nephritis and uveitis syndrome $(n=2,5.13 \%)$. Other diagnoses, found in one patient each, included toxoplasmosis, post-procedural uveitis, sarcoidosis with coexistent collagen vascular disease, idiopathic retinitis, vasculitis, aneurysms, and neuroretinitis (IRVAN), multiple evanescent white dot syndrome (MEWDS), and undifferentiated posterior uveitis. A summary of all diagnoses and their relative frequencies are shown in Table 3. Of the 24 patients with anterior uveitis, ten $(41.67 \%)$ had acute onset, while seven (29.17\%) had chronic onset, and another seven $(29.17 \%)$ experienced an insidious onset. Seven $(29.17 \%)$ of the 24 anterior uveitis patients had bilateral disease and $17(70.83 \%)$ had unilateral disease. Of the seven patients with intermediate uveitis, none had acute onset, while five $(71.43 \%)$ had a chronic onset and two $(28.57 \%)$ had an insidious onset. Five (71.43\%) of the seven intermediate uveitis patients had bilateral disease and two (28.57\%) had unilateral disease. Of the five patients diagnosed with posterior uveitis, none had an acute onset. Three (60.00\%) of the five posterior uveitis patients had a chronic onset and two $(40.00 \%)$ had an insidious onset. Four $(80.00 \%)$ of the five posterior uveitis patients had bilateral disease, while only one $(20.00 \%)$ had unilateral disease. Two $(66.67 \%)$ of the three panuveitis patients had chronic onset and one (33.33\%) had an insidious onset. Two $(66.67 \%)$ of the three panuveitis patients had bilateral disease and only one (33.33\%) had unilateral disease. A summary of uveitis characteristics by site of ocular inflammation is found in Table 1.
Ophthalmic management and interventions were documented. During the follow-up period, 26 (66.67\%) of the 39 patients received local steroids and nine (23.08\%) received systemic steroids. Antimetabolites were prescribed to eight $(20.51 \%)$ of the patients, and only one $(2.56 \%)$ was prescribed an anti-tumor necrosis factor agent. Three $(7.69 \%)$ were given subtenon injections and one $(2.56 \%)$ was given an intravitreal injection. Five (12.82\%) patients who developed glaucoma were managed medically, and two (5.13\%) of those patients underwent glaucoma surgery. Cataract surgery was performed in four (10.26\%) patients, and one $(2.54 \%)$ underwent a pars plana vitrectomy. Ophthalmic management and interventions are summarized in Table 4.

BCVA was recorded at the patients' initial and final visits and converted to $\log$ MAR. The initial $\log$ MAR across all anatomical locations was $0.458 \pm 0.680 \log \mathrm{MAR}$ and the final score was $0.411 \pm 0.760 \log \mathrm{MAR}$. All groups demonstrated an improved $\log$ MAR at the final visit compared with $\log M A R$ at baseline except the anterior uveitis group, which had an initial score of $0.451 \pm 0.713 \log M A R$ and a final score of $0.518 \pm 0.960 \log$ MAR. The biggest improvement in vision occurred in the intermediate uveitis group, which had an initial mean score of $0.592 \pm 0.870 \log$ MAR and a final score of $0.313 \pm 0.491 \log$ MAR. The posterior uveitis group had an initial score of $0.295 \pm 0.329 \log$ MAR and a final score of $0.226 \pm 0.345 \log$ MAR. Finally, the panuveitis group had an initial score of $0.476 \pm 0.511 \log$ MAR and a final score of $0.366 \pm 0.493 \log$ MAR.

Initial and final IOP measurements were also recorded. Overall, initial and final IOP measurements were stable at $14.27 \pm 4.85 \mathrm{mmHg}$ initially and $14.22 \pm 4.25 \mathrm{mmHg}$ at the final visit. Both the anterior and intermediate uveitis groups showed a modest increase in IOP from baseline to the final visit. In the anterior uveitis group, the initial IOP was $14.69 \pm 5.95 \mathrm{mmHg}$ and the final IOP was slightly elevated at $15.59 \pm 4.47 \mathrm{mmHg}$. In the intermediate uveitis group, the initial IOP was $11.83 \pm 3.46 \mathrm{mmHg}$ and the final IOP was $13.58 \pm 3.70 \mathrm{mmHg}$. In the posterior uveitis group, the initial IOP was $15.0 \pm 2.92 \mathrm{mmHg}$ and the final IOP was decreased at $12.0 \pm 3.46 \mathrm{mmHg}$. The initial IOP in the panuveitis group was $16.6 \pm 1.14 \mathrm{mmHg}$, but the final IOP was considerably decreased at $11.8 \pm 3.03 \mathrm{mmHg}$. BCVA and IOP outcomes are summarized in Table 1.

\section{Discussion}

This retrospective study seeks to report and compare the demographics, etiologies, treatments, and outcomes in a population of 39 pediatric uveitis patients at a tertiary referral 


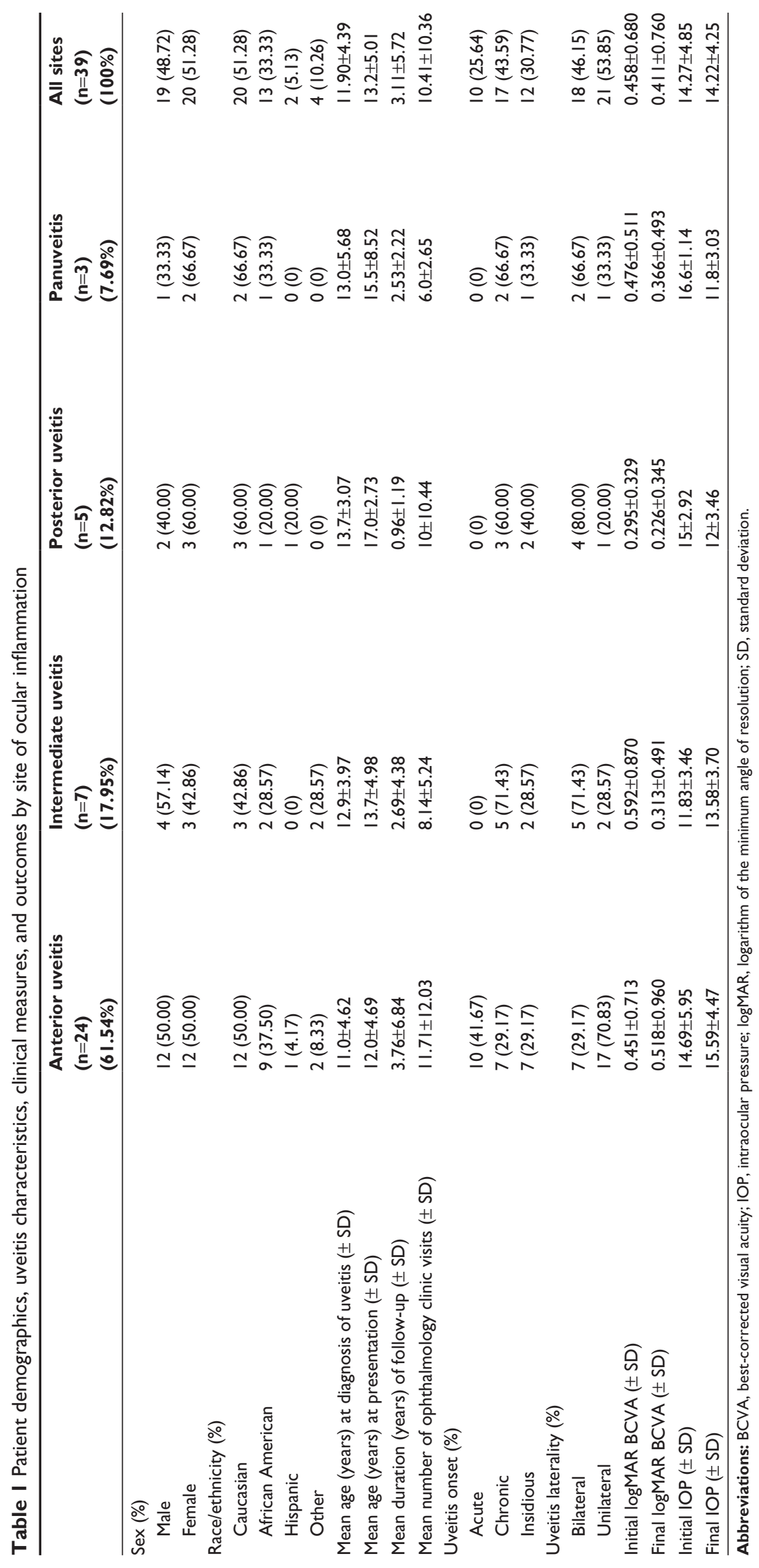


Table 2 Distribution of uveitis subtype

\begin{tabular}{lll}
\hline $\begin{array}{l}\text { Anatomical } \\
\text { localization }\end{array}$ & $\begin{array}{l}\text { Number } \\
\text { of patients (eyes) }\end{array}$ & $\begin{array}{l}\text { Percentage } \\
\text { of patients (eyes) }\end{array}$ \\
\hline Anterior uveitis & $24(3 \mathrm{I})$ & $61.53(54.39)$ \\
Intermediate uveitis & $7(\mathrm{I})$ & $17.95(21.05)$ \\
Posterior uveitis & $5(9)$ & $12.82(15.79)$ \\
Panuveitis & $3(5)$ & $7.69(8.77)$ \\
\hline
\end{tabular}

center in Virginia, USA, with other pediatric uveitis populations published in the literature. A comparison of the current series with other key studies is found in Table 5.

Over a 30-year period, 491 patients with uveitis were seen in the ophthalmology clinic at the University of Virginia. Of those 491 patients, 39 (7.94\%) were pediatric patients diagnosed with uveitis and aged $0-18$ years. This falls within the reported range of $2.1 \%-13.8 \%$ for pediatric uveitis in the total uveitis population reported in the literature. ${ }^{1-3} \mathrm{In}$ older US studies from the 1960 s, the female to male ratio was higher, and thought to be the result of the fact that more girls have JIA than boys. ${ }^{5,12,13}$ The female to male ratio in our study was approximately equal at 1.05:1, a pattern reflected in more recent studies. Two studies in Boston report the percent of female patients to be $53.5 \%$ (in 2005) and 54\% (in 2009). ${ }^{3,8}$ In a Jerusalem study that included patients aged 0-18 years, a 1:1 female to male ratio was found. ${ }^{2}$

Of the 57 eyes included in our study, 31 (54.39\%) had anterior uveitis, $12(21.05 \%)$ had intermediate uveitis, nine $(15.79 \%)$ had posterior uveitis, and five $(8.61 \%)$ had

Table 3 Diagnoses of pediatric uveitis

\begin{tabular}{lll}
\hline Diagnosis & Patients (n) & Patients (\%) \\
\hline Trauma & 10 & 25.64 \\
Anterior uveitis, undifferentiated & 7 & 17.95 \\
Intermediate uveitis, undifferentiated & 6 & 15.38 \\
Herpetic anterior uveitis & 3 & 7.69 \\
HLA-B27-associated anterior & 3 & 7.69 \\
uveitis & & \\
Panuveitis, undifferentiated & 2 & 5.13 \\
TINU & 2 & 5.13 \\
Toxoplasmosis & $\mathrm{I}$ & 2.56 \\
Post-procedural & $\mathrm{I}$ & 2.56 \\
Sarcoidosis with collagen vascular & $\mathrm{I}$ & 2.56 \\
disease & & \\
IRVAN & $\mathrm{I}$ & 2.56 \\
MEWDS & $\mathrm{I}$ & 2.56 \\
Posterior uveitis, undifferentiated & $\mathrm{I}$ & 2.56 \\
Total & 39 & 100 \\
\hline Notes: HLA-B27-assogated
\end{tabular}

Notes: HLA-B27-associated anterior uveitis designation includes psoriatic arthritis $(n=l)$, ulcerative colitis $(n=l)$, unspecified seronegative spondyloarthropathy.

Abbreviations: TINU, tubulointerstitial nephritis and uveitis syndrome; IRVAN, idiopathic retinitis, vasculitis, aneurysms, and neuroretinitis; MEWDS, multiple evanescent white dot syndrome.
Table 4 Management and interventions

\begin{tabular}{ll}
\hline Treatments and interventions & $\mathbf{n ~ ( \% )}$ \\
\hline Local steroids & $26(66.67)$ \\
Systemic steroids & $9(23.08)$ \\
Antimetabolites & $8(20.5 \mathrm{I})$ \\
Anti-TNF agents & $\mathrm{I}(2.56)$ \\
Subtenon injection & $3(7.69)$ \\
Intravitreal injection & $\mathrm{I}(2.56)$ \\
Glaucoma topical treatment & $5(\mathrm{I} 2.82)$ \\
Glaucoma surgery & $2(5.13)$ \\
Cataract surgery & $4(10.26)$ \\
Pars plana vitrectomy & $\mathrm{I}(2.56)$ \\
\hline
\end{tabular}

Abbreviation: TNF, tumor necrosis factor.

panuveitis. These distributions are in line with those reported in similar studies conducted in the USA; ;,7,8 however, there is a significant difference between our distribution and that found at sites outside of the USA..$^{1,2,14,15}$ In one Finnish study, anterior uveitis was found in $90.9 \%$ of cases, intermediate uveitis in $1.8 \%$, posterior uveitis in $23.3 \%$, and panuveitis in $1.8 \% .{ }^{1}$ A study in Jerusalem found anterior uveitis in only $13.4 \%$ of cases. $^{2}$ Studies in Finland (1.8\%) and Australia (5.7\%) found significantly lower percentages of intermediate uveitis compared with our study $(21.05 \%) .{ }^{1,14}$

The distribution of uveitis cases does not only vary geographically, but also has shifted over time even within the USA. ${ }^{12,13,16}$ In a San Francisco study conducted in 1954, anterior uveitis accounted for $31.5 \%$ of cases, intermediate uveitis for $7.5 \%$, posterior uveitis for $49 \%$, and panuveitis for $2 \% .^{12} \mathrm{~A}$ similarly high proportion of posterior uveitis cases (67.7\%) was found in an Ohio study in 1969. ${ }^{16}$

Like subtype distribution, uveitis etiologies vary geographically and historically. In our study, uveitis secondary to trauma was the most frequent cause of uveitis, occurring in ten $(25.64 \%)$ of the 39 patients. Traumatic uveitis has been reported in the literature, but has typically accounted for a smaller percentage of cases. In the study in Jerusalem, ten of 110 non-infectious uveitis cases were traumatic. ${ }^{2}$ Undifferentiated uveitis was found in 16 (41.03\%) of 39 patients, which falls within the wide range reported in the literature. In the Jerusalem study, undifferentiated uveitis accounted for $25.40 \%$ of cases, while in the Netherlands, undifferentiated uveitis accounted for more than half of cases at $53.66 \%{ }^{2,15}$

Only one patient (2.56\%) had toxoplasmosis-associated uveitis, which was slightly lower than in other key US studies, which range from $3.35 \%$ to $7.7 \% .{ }^{3,7,8}$ However, our study had three patients (7.69\%) with HLA-B27-associated anterior uveitis, which is higher than that reported in the literature. Two Boston studies reported HLA-B27-associated anterior uveitis in $0.7 \%-1.86 \%$ of patients. ${ }^{7,8}$ 


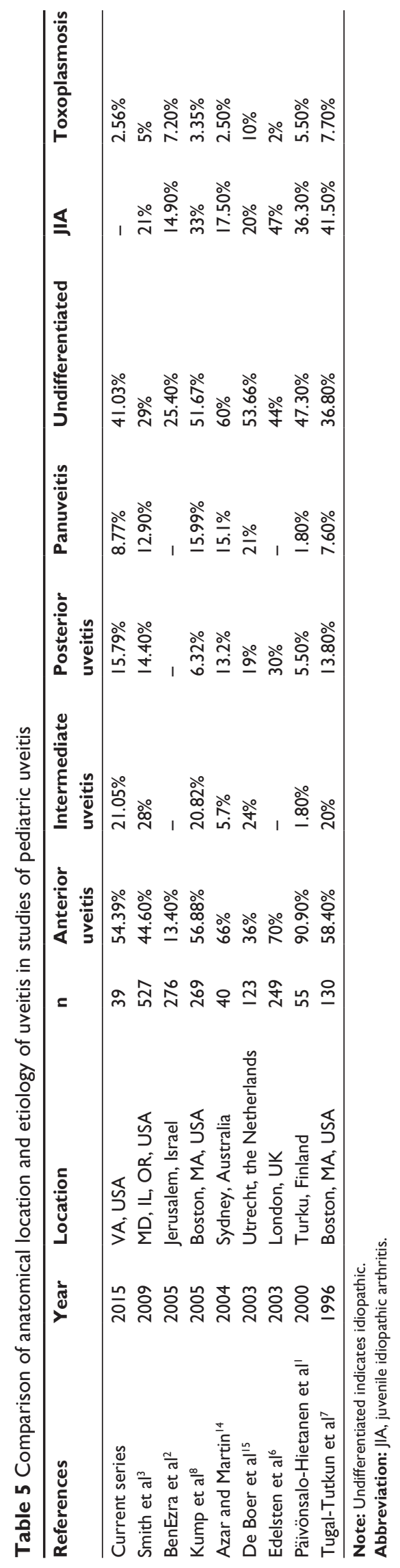

In contrast with many other studies, which focus extensively on JIA, we chose not to include these patients in our analysis because of variable practice and referral patterns in our area over the course of the study period. Recent studies have cited that $14 \%-47 \%$ of cases are attributable to JIA., ${ }^{2,6}$ One study posited that the approximately 50 -fold difference in JIA rates between communities is only partly to do with natural differences and more to do with the different methods of case attainment at different centers and differences in study design, which was the primary reason we chose to exclude of these patients from our analysis. ${ }^{17}$

Patients in our study had a mean duration of follow-up of 3.11 years for all anatomical locations. The anterior uveitis patients had the longest mean follow-up time at 3.76 years, while posterior uveitis patients had the shortest mean follow-up time at only 0.96 years. This likely reflects a tendency for anterior uveitis patients to be referred to ophthalmology sooner due to earlier detection than posterior uveitis patients, which could explain the difference in follow-up times. Furthermore, although we did not find this to be the case in our study, a longer time interval from disease onset to referral in posterior uveitis coupled with involvement of the retina in posterior uveitis mean that pediatric patients with posterior uveitis are more likely to suffer vision loss than patients with other uveitis subtypes. ${ }^{18}$

We investigated the burden of disease by calculating the mean number of ophthalmology clinic visits. The overall number of mean visits was 10.41 across all anatomical locations. Anterior uveitis patients visited the clinic the most often, with a mean of 11.71 visits, and the panuveitis patients visited the least often, with a mean of 6.0 visits. The average age at diagnosis of uveitis in our study was 11.9 years for all anatomical locations. Anterior uveitis had the lowest average age of diagnosis at 11.0 years, while the average ages were 12.9 years for intermediate uveitis, 13.7 years for posterior uveitis, and 13.0 years for panuveitis. A similar pattern of age at diagnosis was seen in one Boston study, although all ages were slightly lower compared with ours. ${ }^{8}$ Part of the difference in age at presentation and diagnosis is due to the fact that we included patients aged $0-18$ years, while many other studies included only patients aged $0-16$ years. However, we believe that it was more appropriate to include patients diagnosed on or before their 18th birthday, which has also been done in the literature previously. ${ }^{2}$

Rates of complications have been discussed in previous studies. Rates of glaucoma in pediatric uveitis patients have been reported from a low of $2 \%$ in undifferentiated uveitis to a high of $30 \%$ in JIA patients. ${ }^{7}$ In our study, five (12.82\%) 
patients developed glaucoma. All were medically managed, and two eventually underwent glaucoma surgery. This rate is somewhat lower than the rates of glaucoma found in other studies, which is likely attributable, at least in part, to the fact that we did not have any JIA patients in our cohort. Cataract surgery was performed in four (10.26\%) patients. These rates are somewhat lower than the rates of complications found in other studies, which is likely due to the fact that some patients had a very short follow-up period and we did not capture the number of all patients who developed cataracts, but instead recorded only those patients who required cataract surgery.

The mean baseline IOP was $14.27 \pm 4.85 \mathrm{mmHg}$, and at final follow-up was $14.22 \pm 4.25 \mathrm{mmHg}$. The posterior and panuveitis groups saw a decrease in IOP at final follow-up, while the anterior and intermediate uveitis groups saw a slight increase in IOP at final follow-up.

Visual acuity improved overall from $0.458 \pm 0.680$ $\log$ MAR at baseline visit to $0.411 \pm 0.760 \log$ MAR at final follow-up. All subgroups saw an improvement in visual acuity at the final follow-up visit, except the anterior uveitis group. In this group, final BCVA was $0.518 \pm 0.960 \log$ MAR compared with $0.451 \pm 0.713 \log M A R$ at baseline. The intermediate uveitis group had the greatest improvement in visual acuity, from $0.592 \pm 0.870 \log$ MAR at baseline to $0.313 \pm 0.491$ $\log$ MAR at final follow-up. It has been reported that up to one-third of pediatric uveitis patients develop severe vision impairment as a result of complications of uveitis, ${ }^{4}$ however, this was not found in our study, suggesting the importance of prompt referral to and careful management of these patients by uveitis specialists to prevent poor visual outcomes.

The major weaknesses of this study include the limited sample size and the retrospective study design, both of which make the study susceptible to referral, treatment, and data collection biases. Because no standardized follow-up period was required for inclusion in the study, it is possible that the visual and IOP outcomes represent only patients with more severe disease or those with longer follow-up periods. Owing also to the 30-year span of this study, patients were seen by different ophthalmologists. Because of this, and because of the fact that some of the data was taken from a time prior to efforts to standardize the uveitis nomenclature, there was possibly some discrepancy in categorization of uveitis subtype over time. Additionally, lack of a uveitis specialist to make precise diagnoses at various times during the 30 -year period may have influenced the diagnoses and management of patients. Due to the above limitations, the statistical analysis was descriptive in nature.
In conclusion, our study demonstrates that while non-JIA pediatric uveitis can be difficult to diagnose and manage due to the number of uveitis etiologies present in childhood, many cases may be due to trauma and respond to conservative interventions. This study also highlights the burdensome nature of the uveitides and the importance of regular referral to a uveitis specialist to prevent long-term ocular damage and vision loss. We found that BCVA and IOP did not vary significantly during the period of follow-up, suggesting that close management by an ophthalmologist may prevent adverse visual outcomes.

\section{Disclosure}

The authors report no conflicts of interest in this work.

\section{References}

1. Päivönsalo-Hietanen T, Tuominen J, Matti Saari K. Uveitis in children population-based study in Finland. Acta Ophthalmol. 2000;78(1):84-88.

2. BenEzra D, Cohen E, Maftzir G. Uveitis in children and adolescents Br J Ophthalmol. 2005;89(4):444-448.

3. Smith JA, Mackensen F, Sen HN, et al. Epidemiology and course of disease in childhood uveitis. Ophthalmology. 2009;116(8):1544-1551.

4. Cunningham ET. Uveitis in children. Ocul Immunol Inflamm. 2000;8(4): 251-261.

5. Holland GN, Stiehm ER. Special considerations in the evaluation and management of uveitis in children. Am J Ophthalmol. 2003;135(6):867-878.

6. Edelsten C, Reddy MA, Stanford MR, Graham EM. Visual loss associated with pediatric uveitis in English primary and referral centers. Am J Ophthalmol. 2003;135(5):676-680.

7. Tugal-Tutkun I, Havrlikova K, Power WJ, Foster CS. Changing patterns in uveitis of childhood. Ophthalmology. 1996;103(3):375-383.

8. Kump LI, Cervantes-Castañeda RA, Androudi SN, Foster CS Analysis of pediatric uveitis cases at a tertiary referral center. Ophthalmology. 2005;112(7):1287-1292.

9. Standardization of Uveitis Nomenclature (SUN) Working Group. Standardization of uveitis nomenclature for reporting clinical data. Results of the First International Workshop. Am J Ophthalmol. 2005; 140(3):509-516.

10. Lange C, Feltgen N, Junker B, Schulze-Bonsel K, Bach M. Resolving the clinical acuity categories "hand motion" and "counting fingers" using the Freiburg Visual Acuity Test (FrACT). Graefes Arch Clin Exp Ophthalmol. 2009;247(1):137-142.

11. Kwon YH, Kim CS, Zimmerman MB, Alward WL, Hayreh SS. Rate of visual field loss and long-term visual outcome in primary open-angle glaucoma. Am J Ophthalmol. 2001;132(1):47-56.

12. Kimura S, Hogan MJ, Thygeson P. Uveitis in children. AMA Am J Dis Child. 1954;87(1):40-48.

13. Perkins ES. Pattern of uveitis in children. Br J Ophthalmol. 1966;50: 169-185.

14. Azar D, Martin F. Paediatric uveitis: a Sydney clinic experience. Clin Experiment Ophthalmol. 2004;32(5):468-471.

15. De Boer J, Wulffraat N, Rothova A. Visual loss in uveitis of childhood. Br J Ophthalmol. 2003;87(7):879-884.

16. Makley TA, Long J, Suic T. Uveitis in children. J Pediatr Ophthalmol. 1966;50:169-185.

17. Manners PJ, Bower C. Worldwide prevalence of juvenile arthritis: why does it vary so much? J Rheumatol. 2002;29(7):1520-1530.

18. Thadani SM, Foster CS. Treatment of ocular inflammation in children. Paediatr Drugs. 2004;6(5):289-301. 


\section{Publish your work in this journal}

Clinical Ophthalmology is an international, peer-reviewed journal covering all subspecialties within ophthalmology. Key topics include: Optometry; Visual science; Pharmacology and drug therapy in eye diseases; Basic Sciences; Primary and Secondary eye care; Patient Safety and Quality of Care Improvements. This journal is indexed on

Submit your manuscript here: http://www.dovepress.com/clinical-ophthalmology-journal
PubMed Central and CAS, and is the official journal of The Society of Clinical Ophthalmology (SCO). The manuscript management system is completely online and includes a very quick and fair peer-review system, which is all easy to use. Visit http://www.dovepress.com/ testimonials.php to read real quotes from published authors. 